\title{
Cost-Effectiveness and Budget Impact of Lumacaftor/lvacaftor in the Treatment of Cystic Fibrosis
}

\author{
Pratyusha Vadagam, MS; Khalid M. Kamal, MPharm, PhD; Jordan R. Covvey, PharmD, PhD, BCPS; \\ Vincent Giannetti, PhD; and Kumar Mukherjee, PhD
}

\begin{abstract}
BACKGROUND: Cystic fibrosis (CF) is a chronic, progressive, genetic disease affecting more than 30,000 people in the United States and 70,000 people globally. The goals of treatment are to slow disease progression, reduce pulmonary exacerbations, relieve chronic symptoms, and improve the patient's quality of life. Lumacaftor/ivacaftor is a new therapy for CF that has demonstrated good clinical outcomes, including improved absolute percentage predicted forced expiratory volume in 1 second $\left(\mathrm{FEV}_{1} \%\right)$. However, given the high cost of therapy, there is a need to evaluate the overall value of lumacaftor/ivacaftor in CF management.
\end{abstract}

OBJECTIVES: To (a) conduct a cost-effectiveness analysis (CEA) of lumacaftor/ivacaftor to understand the overall effectiveness of the drug compared with its costs and (b) conduct a budget impact analysis (BIA) to understand the potential financial effect of introducing a new drug in a health plan.

METHODS: Two static decision models were developed using Microsoft Excel to evaluate the cost-effectiveness and budget impact of lumacaftor/ ivacaftor over a 1-year time frame from a payer perspective. Model inputs included drug costs (wholesale acquisition costs), drug monitoring schedules (package inserts), drug monitoring costs (Centers for Medicare \& Medicaid physician fee schedule and published literature), $\mathrm{FEV}_{1} \%$ predicted and pulmonary exacerbation values (clinical trials), and cost to treat pulmonary exacerbations (published literature). The outcomes in the CEA included total cost of therapy; average cost-effectiveness ratio (ACER), defined as cost per $\mathrm{FEV}_{1} \%$ predicted; and incremental cost-effectiveness ratio (ICER), defined as the difference in the ratio of cost per $\mathrm{FEV}_{1} \%$ predicted of lumacaftor/ivacaftor and placebo. Outcomes in the BIA included total budget impact; cost per member per month (PMPM), defined as total budget impact per hypothetical plan population; and cost per treated member per month (PTMPM), defined as total budget impact per target CF population. All costs were adjusted to 2016 dollars, and one-way sensitivity analyses were conducted to test the model robustness given uncertainty in model inputs and study assumptions.

RESULTS: The annual cost of therapy per patient for lumacaftor/ivacaftor was $\$ 379,780$. The ACER for lumacaftor/ivacaftor was $\$ 151,912$, while the ICER for lumacaftor/ivacaftor compared with placebo was $\$ 95,016$ per $\mathrm{FEV}_{1} \%$ predicted. The annual total budget impact due to the inclusion of lumacaftor/ivacaftor on the health plan formulary was $\$ 266,046$. The PMPM cost was $\$ 0.02$ and the PTMPM cost was $\$ 620.67$.

CONCLUSIONS: In patients with CF, lumacaftor/ivacaftor has demonstrated better clinical effectiveness compared with placebo alongside an increased drug acquisition cost. However, the therapy may be a viable alternative to existing standard therapy over a short time horizon. Health care payers, both private and public, need to evaluate the cost-effectiveness and the financial effect when considering expansion of new drug coverage in $\mathrm{CF}$ management.

\section{J Manag Care Spec Pharm. 2018;24(10):987-97}

Copyright $\odot 2018$, Academy of Managed Care Pharmacy. All rights reserved.

\section{What is already known about this subject}

Lumacaftor/ivacaftor was approved by the U.S. Food and Drug Administration in 2015.

Lumacaftor/ivacaftor has been rejected by international health technology assessments such as the National Institute for Health and Care Excellence of the United Kingdom and the National Centre for Pharmacoeconomics of Ireland because of its poor costeffectiveness profile and high annual treatment cost per patient.

\section{What this study adds}

Incremental cost-effectiveness ratio (ICER) was used as cost per absolute percentage predicted forced expiratory volume in 1 second $\left(\mathrm{FEV}_{1} \%\right)$ instead of cost per quality-adjusted life-years (QALY), which is reported in other health technology assessments. This study used $\mathrm{FEV}_{1} \%$ predicted from the clinical trial because the long-term clinical effectiveness and the transition states possible after treatment initiation were not known.

In patients with cystic fibrosis and with homozygous F508del mutation, lumacaftor/ivakaftor had an ICER of $\$ 95,016$ per $\mathrm{FEV}_{1} \%$ predicted compared with placebo.

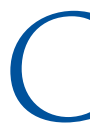
ystic fibrosis (CF) is a chronic, progressive, genetic disease that primarily affects the respiratory, digestive, and reproductive organ systems in children and adults. ${ }^{1}$ It is primarily characterized by abnormally thick mucus linings in the lungs resulting in reduced function and development of potentially fatal respiratory infections. ${ }^{2}$ The disease is inherited in an autosomal recessive pattern and is caused by various mutations in cystic fibrosis transmembrane conductance regulator (CFTR) gene, which affects the composition of the mucous layer lining the epithelial cells in the lungs and pancreas. ${ }^{3}$ This leads to disruption of ion transport and changes in the amount of water and texture of mucus in cell linings of the lungs. ${ }^{3}$ In addition to being associated with respiratory dysfunction, CF also affects the digestive system and reproductive system, hastening disease progression and shortening life expectancy., ${ }^{4,5}$

$\mathrm{CF}$ is a relatively uncommon diagnosis, with an estimated prevalence of 30,000 people in the United States and more than 70,000 people globally in 2013. ${ }^{2}$ In the United States, approximately 1,000 new cases of CF are diagnosed each year with more than $75 \%$ of individuals diagnosed by 2 years of 
age. ${ }^{2}$ Severity of illness is assessed by the absolute percentage predicted forced expiratory volume in 1 second $\left(\mathrm{FEV}_{1} \%\right.$ predicted), with $>70 \%$ categorized as mild, $40 \%-69 \%$ as moderate, and $<40 \%$ as severe. ${ }^{6}$

Despite a low prevalence, the costs of illness are significant. In 2011, the annual estimated average costs of CF care were $\$ 30,000, \$ 57,000$, and $\$ 215,000$ for patients with mild, moderate, and severe disease, respectively. Costs were reported to be the highest among children aged 10-14 years and lowered with increasing age through 45 years across all 3 disease severity groups. Specifically, severe disease in patients aged 10-14 years incurred a much higher annual cost of care $(\$ 343,900)$ when compared with mild disease in patients aged $40-44$ years $(\$ 15,600)^{7}$

There is no definitive cure for $\mathrm{CF}$, so the goals of current therapy focus on slowing disease progression, reducing pulmonary exacerbations, relieving chronic symptoms, and improving the patient's quality of life. ${ }^{8}$ Existing treatments for the pulmonary manifestations of CF include broad maintenance therapies that help to clear mucus from the lungs and to prevent or treat pulmonary infections. However, in recent years, new treatments for CF have been introduced. The U.S. Food and Drug Administration (FDA) approved a CFTR modulator therapy called ivacaftor (Kalydeco) in 2012 and later in 2015 as lumacaftor/ivacaftor. ${ }^{9,10}$ Ivacaftor is indicated in patients aged 6 years and older who have the G551D mutation in the CFTR gene, which includes approximately $4 \%$ of patients with CF. Lumacaftor/ivacaftor is indicated in patients aged 6 years and older who have the F508del mutation in the CFTR gene, including approximately 45\% of patients, and significantly expands treatment utility.

Two major phase 3 clinical trials of lumacaftor/ivacaftor, TRAFFIC and TRANSPORT, demonstrated significant improvements in $\mathrm{FEV}_{1} \%$ predicted values from baseline among patients receiving the drug-ranging from 2.6 to 3 units change in $\mathrm{FEV}_{1} \%$ predicted across the studies and in the pooled analysis. ${ }^{10-12}$ Data from the pooled analysis also demonstrated a $30 \%$ to $39 \%$ reduction in the rate of pulmonary exacerbations among patients who received lumacaftor/ ivacaftor compared with placebo. ${ }^{13}$ Although lumacaftor/ ivacaftor has demonstrated higher efficacy, lower side effects, and, most importantly, has offered a new treatment option to patients with CF with the F508del mutation, the estimated wholesale acquisition cost of $\$ 270,172.77$ in 2016 has become a source of concern for health care stakeholders. ${ }^{14}$ Despite the clinical benefits, the high cost of therapy necessitates an assessment of the overall value of the drug in terms of its cost-effectiveness and the financial impact on health plans.

Since the launch of lumacaftor/ivacaftor, several health technology assessments (HTA) have provided additional data. In 2015, the National Centre for Pharmacoeconomics (NCPE) of Ireland compared lumacaftor/ivacaftor with the standard of care and estimated an incremental quality-adjusted life-years (QALY) gain of 2.45 at an incremental cost of $€ 903,947$ (U.S. $\$ 1,017,103.08$ in 2016). The incremental cost-effectiveness ratio (ICER) was estimated at $€ 369,141$ per QALY (U.S. $\$ 415,350$ in 2016). ${ }^{15}$ In 2016, the National Institute for Health and Care Excellence (NICE) of the United Kingdom also issued a draft guidance against recommending lumacaftor/ivacaftor for treating CF, citing a high annual treatment cost of $€ 104,000$ (U.S. $\$ 118,560$ in 2016) per patient as the reason for its decision. Similarly, the Scottish Medicines Consortium did not recommend adoption of lumacaftor/ivacaftor. ${ }^{16}$ Despite these assessments, little information is available relevant to decision making in the United States, and there is a dearth of evidence from published literature regarding the drug's cost-effectiveness and affordability. Thus, it is important to assess the cost-effectiveness and budget impact for lumacaftor/ivacaftor from a U.S. health care payer perspective.

This study's objectives were to (a) conduct a cost-effectiveness analysis (CEA) of lumacaftor/ivacaftor to understand the overall effectiveness of the drug compared with its costs and (b) conduct a budget impact analysis (BIA) to understand the potential financial effect of introducing a new drug in a health plan.

\section{Methods}

\section{Model Characteristics}

Two static decision models were developed using Microsoft Excel 2013 (Microsoft, Redmond, WA) to evaluate the costeffectiveness and budget impact of lumacaftor/ivacaftor in a hypothetical CF population. Both models were analyzed over a 1-year time frame. A shorter time frame was chosen to be consistent with the length of phase 3 clinical trials and the outcomes data from the trials. ${ }^{17}$ In addition, there was a lack of data on long-term clinical efficacy of the drug at the time of the study. Data up to 96 weeks from the phase 3 PROGRESS trial are available, which followed treatment and placebo groups from phase 3 TRAFFIC and TRANSPORT trials. While the treatment group in the trial continued on lumacaftor/ivacaftor from week 24 to week 96, the placebo group rolled over from no treatment to different doses of lumacaftor/ivacaftor after 24 weeks. Also, the primary outcome of this trial was long-term safety of the combined therapy, and the treatment groups were compared with a cohort of matched control patients who were also homozygous for the F508del-CFTR mutation from the Cystic Fibrosis Foundation Patient Registry. ${ }^{18}$ These reasons necessitated using a shorter time horizon and static decision models.

This study was analyzed from a U.S. third-party payer perspective and direct costs such as drug acquisition costs, monitoring costs, and costs to treat adverse events were included in the analyses.

The hypothetical patient population in both analyses was CF patients aged 12 years and older with homozygous F508del mutation of the CFTR gene who were on standard therapy at baseline and treated by lumacaftor/ivacaftor. The demographics 
TABLE 1 Safety and Efficacy of Lumacaftor/Ivacaftor from Phase 3 and Published Clinical Trials ${ }^{11,12}$

\begin{tabular}{|c|c|c|c|c|c|c|}
\hline & \multicolumn{2}{|c|}{ TRAFFIC 11} & \multicolumn{2}{|c|}{ TRANSPORT $^{12}$} & \multicolumn{2}{|c|}{ Pooled Analysis } \\
\hline & $\begin{array}{l}\text { Placebo } \\
(\mathrm{n}=184)\end{array}$ & $\begin{array}{l}\text { Lumacaftor/ } \\
\text { Ivacaftor } \\
(\mathrm{n}=182)\end{array}$ & $\begin{array}{l}\text { Placebo } \\
(\mathrm{n}=187)\end{array}$ & $\begin{array}{l}\text { Lumacaftor/ } \\
\text { Ivacaftor } \\
(\mathbf{n}=187)\end{array}$ & $\begin{array}{l}\text { Placebo } \\
(\mathrm{n}=371)\end{array}$ & $\begin{array}{l}\text { Lumacaftor/ } \\
\text { Ivacaftor } \\
(\mathbf{n}=369)\end{array}$ \\
\hline \multicolumn{7}{|l|}{ Efficacy at week 24} \\
\hline $\begin{array}{l}\text { Absolute change in } \mathrm{FEV}_{1} \text { from baseline, } \\
\text { mean (SE) }\end{array}$ & $-0.44 \quad(0.52)$ & $2.16 \quad(0.53)$ & $-0.15 \quad(0.54)$ & $2.85 \quad(0.54)$ & $-0.29(0.53)$ & $2.50 \quad(0.53)$ \\
\hline $\begin{array}{l}\text { Percentage difference vs. placebo in } \\
\text { absolute change, mean }(95 \% \mathrm{CI})\end{array}$ & - & $2.6(1.2-4.0)$ & - & $3.0(1.6-4.4)$ & - & $2.8(1.8-3.8)$ \\
\hline \multicolumn{7}{|l|}{ Safety at week 48} \\
\hline $\begin{array}{l}\text { Participants affected or at risk for } \\
\text { pulmonary exacerbations, } \mathrm{n}(\%)\end{array}$ & $112(60.86)^{10}$ & $\begin{array}{ll}73 & (40.10)^{10}\end{array}$ & $139(74.33)^{10}$ & $79 \quad(42.24)^{10}$ & $182(49.2)^{17, a}$ & $132(35.8)^{17, a}$ \\
\hline $\begin{array}{l}\text { Participants discontinued because of } \\
\text { an adverse event, } \mathrm{n}(\%)\end{array}$ & - & - & - & - & $\begin{array}{ll}6 & (1.6)^{17, a}\end{array}$ & $(4.6)^{17, a}$ \\
\hline
\end{tabular}

and clinical characteristics of the study population were based on clinical trial data of lumacaftor/ivacaftor. ${ }^{17}$

Lumacaftor/ivacaftor does not replace any existing therapy, since the drug targets a specific gene mutation. Thus, the CEA compared lumacaftor/ivacaftor with placebo, although both groups were on standard therapy consisting of maintenance therapies at baseline. The budget impact was calculated based on preinclusion and postinclusion of the drug in the formulary consisting of standard therapy, including pulmonary medications such as bronchodilators, inhaled antibiotics, dornase alfa, inhaled hypertonic saline, and inhaled corticosteroids. ${ }^{17}$

The outcomes of interest for the CEA included cost of therapy per patient; average cost-effectiveness ratio (ACER), presented as cost per $\mathrm{FEV}_{1} \%$ predicted per patient; and ICER, presented as additional cost per 1-unit increase in $\mathrm{FEV}_{1} \%$ predicted per patient. The average ratio is particularly useful if the new treatment does not have any comparators, and the adoption of a new treatment is based on its cost-effectiveness profile compared with a placebo/standard therapy. The incremental ratio summarizes the cost-effectiveness of a new treatment over its comparators by considering cost and outcomes. When multiple comparators are available, ACER may not be fully useful and instead ICER is considered. ${ }^{19}$ Given that the comparators are limited for lumacaftor/ivacaftor, both ACER and ICER were calculated using the placebo group as the comparator. The total cost of therapy was calculated as the sum of all costs, including drug costs, monitoring costs, and costs to treat adverse events involved in placebo or lumacaftor/ivacaftor treatment.

The ACER was calculated as total cost of therapy per $\mathrm{FEV}_{1} \%$ predicted at week 24 derived from a pooled analysis of the phase 3 clinical trials. The ICER was calculated as (Cost lumacaftorivacaftor - Cost $\left._{\text {Placebo }}\right) /\left(\right.$ Outcome $_{\text {lumacaftor/ }}$ ivacaftor-Outcome Placebo $\left._{\text {o }}\right)$, where the outcome is $\mathrm{FEV}_{1} \%$ predicted at week 24 from a pooled analysis of the phase 3 clinical trials.

The outcomes of interest for the BIA included total budget impact, per member per month (PMPM) cost, and per treated member per month (PTMPM) cost. The total budget impact was calculated as the difference in budget preinclusion and postinclusion of lumacaftor/ivacaftor to the health plan formulary. The PMPM cost was calculated as total budget impact divided by total hypothetical plan population (1 million) divided by 12 . The PTMPM cost was calculated as total budget impact divided by total target population (35.72) divided by 12 .

\section{Efficacy Inputs}

The $\mathrm{FEV}_{1} \%$ predicted value was used as the efficacy input in the CEA model as it was the most commonly reported primary outcome in the phase 3 clinical trials of lumacaftor/ivacaftor (Table 1). Mean absolute change in $\mathrm{FEV}_{1} \%$ predicted, reported at Week 24 in the clinical trials, was used in the analyses. The safety data on the drug included the most common adverse events reported by subjects in the phase 3 clinical trials. The most common adverse events were defined as those that occurred in at least $10 \%$ of patients in any treatment group. ${ }^{17}$

\section{Cost Inputs}

Drug Acquisition Costs. The drug acquisition costs, reported as the wholesale acquisition cost, were taken from the 2015 RED BOOK dosage information from FDA package inserts. Number of doses per package from RED BOOK were used to calculate the number of packages required per patient per year. For both analyses, the drug acquisition cost was calculated as the product of the package cost and the number of packages required per year. Product rebates, discounts, patients' copay or coinsurance were not considered in the analyses. The dose, 
TABLE 2 Dosing and Acquisition Costs of Lumacaftor/lvacaftor and Standard Therapy 10,17

\begin{tabular}{|c|c|c|c|c|c|}
\hline Drug & Dose, Frequency, Route & Doses per Pack & $\begin{array}{c}\text { Wholesale } \\
\text { Acquisition Price } \\
\text { per Package (\$) }\end{array}$ & $\begin{array}{c}\text { Packages Needed } \\
\text { per Year }\end{array}$ & $\begin{array}{c}\text { Wholesale } \\
\text { Acquisition Price } \\
\text { per Year } 2016(\$)\end{array}$ \\
\hline $\begin{array}{l}\text { Lumacaftor/ivacaftor } \\
\text { (Orkambi) }^{\mathrm{a}}\end{array}$ & $\begin{array}{l}400 \mathrm{mg} \text { lumacaftor }+250 \mathrm{mg} \text { ivacaftor } \\
\text { Q12 hours PO12 hours }\end{array}$ & 112.0 & $19,923.08$ & 13.03 & $270,172.77$ \\
\hline $\begin{array}{l}\text { Bronchodilator } \\
\text { Albuterol } \\
\text { (Proventil HFA)39,b }\end{array}$ & $\begin{array}{l}0.18 \mathrm{mg} \\
\text { Q6 hours inhalation }\end{array}$ & $74,444.0$ & 75.36 & 0.04 & 2.93 \\
\hline $\begin{array}{l}\text { Inhaled antibiotic } \\
\text { Tobramycin } \\
\text { (Tobi) } 40\end{array}$ & $\begin{array}{l}300 \mathrm{mg} \\
\text { Q12 hours inhalation }\end{array}$ & 56.0 & $3,604.00$ & 13.00 & $46,852.00$ \\
\hline $\begin{array}{l}\text { Mucolytic agent } \\
\text { Dornase alfa } \\
\text { (Pulmozyme) }^{41}\end{array}$ & $\begin{array}{l}2.5 \mathrm{mg} \\
\text { Q daily inhalation }\end{array}$ & 30.0 & $3,283.41$ & 12.16 & $39,926.26$ \\
\hline $\begin{array}{l}\text { Mucolytic agent } \\
\text { Hypertonic saline } \\
\text { (NebuSal) }^{42}\end{array}$ & $\begin{array}{l}10 \mathrm{ml} \text { of } 6 \% \text { solution } \\
\text { Q12 hours inhalation }\end{array}$ & 24.0 & 40.00 & 30.40 & $1,216.00$ \\
\hline $\begin{array}{l}\text { Inhaled corticosteroid } \\
\text { Fluticasone } \\
\text { (Flonase) } 43, \mathrm{c}\end{array}$ & $\begin{array}{l}200 \text { mcg } \\
\text { Q daily inhalation }\end{array}$ & 9.9 & 11.84 & 147.40 & $1,745.21$ \\
\hline \multicolumn{6}{|c|}{ 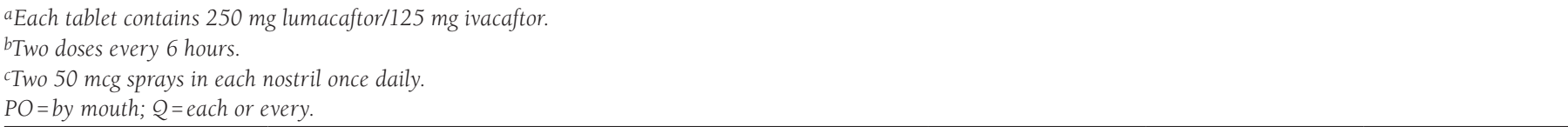 } \\
\hline
\end{tabular}

frequency, route of administration, and acquisition cost of lumacaftor/ivacaftor and standard therapy are presented in Table 2.

Monitoring Costs. The monitoring schedules used in both analyses were taken from the package insert of lumacaftor/ ivacaftor and CF care guidelines reported by the 2013 CF Foundation Patient Registry data. ${ }^{10,20}$ There were no administration costs included in the analyses, since the drugs were administered either by mouth or inhalation. Monitoring test schedules associated with lumacaftor/ivacaftor included aspartate aminotransferase test (AST), alanine aminotransferase test (ALT), and bilirubin, which were taken from the package insert. All other laboratory tests were taken from guidelines published by the CF Foundation. Frequency of clinic visits, outpatient visits, and inpatient stays were taken from published literature. ${ }^{10,20}$ The costs for laboratory and monitoring tests were obtained from the Centers for Medicare \& Medicaid (CMS) 2016 Physician Fee Schedule using the Current Procedural Terminology codes, 2016 Healthcare Bluebook, and from published literature. ${ }^{21,22}$ The drug monitoring test schedules and annual costs are presented in Table 3.

Cost to Treat Adverse Events. Common adverse events were defined as those that occurred in at least 10\% of patients in any treatment group. Pulmonary exacerbation was the most commonly reported serious adverse event in the phase 3 clinical trials of lumacaftor/ivacaftor, and the cost to treat the adverse event was taken from published literature (Table 3). ${ }^{17,23}$ The probabilities of medical events and adverse events were calculated based on the event rate in each group (Appendix A, available in online article). All costs were adjusted to 2016 U.S. dollars using the medical care (drug costs) and medical care services (monitoring costs) components of the Consumer Price Index..24

\section{Model Assumptions}

Several assumptions were made in the study and are summarized as follows:

1. A recently published study reported that the mean weighted medication possession ratio of the CF population for lumacaftor/ivacaftor was $92.28 \%$ with a range of $87 \%-99.5 \% .^{25}$ To provide a fair comparison and avoid preference for lumacaftor/ivacaftor therapy, adherence to lumacaftor/ivacaftor, placebo (CEA), and standard therapy (BIA) were assumed to be $100 \%$.

2. The prevalence of $\mathrm{CF}$ was based on the latest estimates available from 2015 CF Foundation data, which were assumed to be stable in 2016 .

3. Patients were assumed to be maintained on the assigned therapy throughout the duration of the model.

4. The clinical inputs such as $\mathrm{FEV}_{1} \%$ predicted values and pulmonary exacerbation rates taken from published phase 3 clinical trials were assumed to be reliable and valid.

5. Because the analyses had a short analytic time frame, it was assumed that both treatment groups did not undergo a transition to lung transplantation or death. This was based on the adverse events reported in the phase 3 clinical trials, which included pulmonary exacerbations and other minor 


\section{TABLE 3 Cost of Drug Monitoring and Adverse Events}

\begin{tabular}{|c|c|c|c|c|c|}
\hline & Probability & CPT Code & Frequency & Unit Cost $2016(\$)$ & Annual Cost $2016(\$)$ \\
\hline \multicolumn{6}{|l|}{ Drug monitoring } \\
\hline AST & 1.00 & $84450^{44}$ & 4 & $14.00^{45}$ & 56.00 \\
\hline ALT & 1.00 & $84460^{46}$ & 4 & $14.00^{47}$ & 56.00 \\
\hline Bilirubin & 1.00 & 8224748 & 4 & $13.00^{49}$ & 52.00 \\
\hline Sputum/throat cultures & 1.00 & $87070,87205^{50}$ & 4 & 158.4051 & 633.60 \\
\hline PFT & 1.00 & $94010^{52}$ & 2 & $36.52^{53}$ & 73.04 \\
\hline Flu shot & 1.00 & $90656^{54}$ & 1 & $24.00^{55}$ & 24.00 \\
\hline Fat-soluble vitamin blood levels & 1.00 & $\begin{array}{l}\text { A: } 84590^{56} \\
\text { D: } 82306^{57} \\
\text { E: } 84446^{58} \\
\text { K: } 8459759\end{array}$ & 2 & $\begin{array}{l}\text { A: } 31.00^{60} \\
\text { D: } 100.00^{61} \\
\text { E: } 37.00^{62} \\
\text { K: } 36.00^{63}\end{array}$ & 408.00 \\
\hline OGT & 1.00 & $82951,82952^{64}$ & 1 & $34.00^{65}$ & 34.00 \\
\hline Clinic visits & $0.56^{\mathrm{a}}$ & $\begin{array}{c}99205 \text { (initial) } \\
99215 \text { (subsequent) } 66\end{array}$ & 4.3967 & $562.50^{\mathrm{b}}$ & $1,382.85$ \\
\hline Outpatient visits & $0.55^{\mathrm{a}}$ & & $6.26^{67}$ & $291.18^{\mathrm{b}}$ & $1,002.53$ \\
\hline Inpatient stays & $0.28^{\mathrm{a}}$ & & 0.6467 & $7,377.05^{b}$ & $1,321.96$ \\
\hline \multicolumn{6}{|l|}{ Adverse events } \\
\hline Pulmonary exacerbations in placebo group & $0.39^{c}$ & & $3.6^{68}$ & $13,612.65^{23}$ & $19,112.14$ \\
\hline Pulmonary exacerbations in lumacaftor/ivacaftor group & $0.31^{\mathrm{c}}$ & & $3.6^{68}$ & $13,612.65^{23}$ & $15,191.71$ \\
\hline \multicolumn{6}{|c|}{$\begin{array}{l}{ }^{a} \text { Calculated using rate of medical events from O'Sullivan et al. }(2011) .67 \\
\text { bCalculated using Medical Expenditure Panel Survey } 2014 \text { data. }{ }^{69} \\
{ }^{c} \text { Calculated using rate of pulmonary exacerbations in placebo group and lumacaftor/ivacaftor group reported in phase } 3 \text { clinical trial. }{ }^{12} \\
\text { AST=aspartate aminotransferase test; ALT=alanine aminotransferase test; CPT=Current Procedural Terminology; PFT=pulmonary lung function test; OGT=oral } \\
\text { glucose tolerance test. }\end{array}$} \\
\hline
\end{tabular}

events such as cough, increased sputum, headache, hemoptysis, and nasopharyngitis. ${ }^{17}$ This assumption may not be relevant to studies with a longer time horizon.

6. In the CEA, subjects were assumed to be on standard therapy at baseline, which included maintenance drugs.

7. Because lumacaftor/ivacaftor is a novel CFTR modulator therapy, budget impact was calculated to understand the financial effect on a health plan based on preinclusion and postinclusion of the drug on the formulary. The assumption was that the patients continued to receive existing standard therapy consisting of pulmonary medications such as bronchodilators, inhaled antibiotics, dornase alfa, inhaled hypertonic saline, and inhaled corticosteroids before and after the introduction of lumacaftor/ivacaftor.

8. For both analyses, cost due to medical events including clinic visits, outpatient visits, and inpatient stays were assumed to decline by $10 \%$ after the initiation of lumacaftor/ ivacaftor therapy because there were no negative outcomes observed in clinical trials after the initiation of lumacaftor/ ivacaftor. Although counterintuitive given the positive outcomes, 2 directional sensitivity analyses were conducted for these medical events to test the worst-case and bestcase scenarios. The cost of pulmonary exacerbations was adjusted based on the probabilities obtained from published clinical trials.

\section{Sensitivity Analysis}

To account for uncertainty in study variables, univariate or oneway sensitivity analyses were conducted to determine the impact of varying the model inputs in the CEA and BIA of lumacaftor/ ivacaftor. Drug acquisition costs, drug monitoring costs, cost to treat pulmonary exacerbations, and inflation rates were varied over a range of estimates $( \pm 25 \%)$ to test the robustness of the results based on the ranges used in the literature..$^{26-28}$

\section{Results}

Base-Case Analysis: Cost-Effectiveness of Lumacaftor/Ivacaftor The total cost of therapy per patient-defined as the sum of all the costs including drug costs, monitoring costs, and costs to treat adverse events in the lumacaftor/ivacaftor group-was $\$ 379,780$ compared with $\$ 113,735$ in the placebo group. The ACER for lumacaftor/ivacaftor was $\$ 151,912$, indicating a positive change in $\mathrm{FEV}_{1} \%$ predicted value from baseline. The ACER for placebo was $-\$ 392,188$. The estimated ICER of lumacaftor/ ivacaftor over placebo was $\$ 95,016$, indicating the additional cost of a 1-unit positive change in $\mathrm{FEV}_{1} \%$ predicted value by lumacaftor/ivacaftor compared with the placebo group.

Sensitivity Analysis: Cost-Effectiveness of Lumacaftor/Ivacaftor After a $\pm 25 \%$ change in the cost of lumacaftor/ivacaftor, the drug had an ICER ranging from $\$ 70,894$ to $\$ 119,139$. Similarly, lumacaftor/ivacaftor was sensitive to changes in inflation rate 


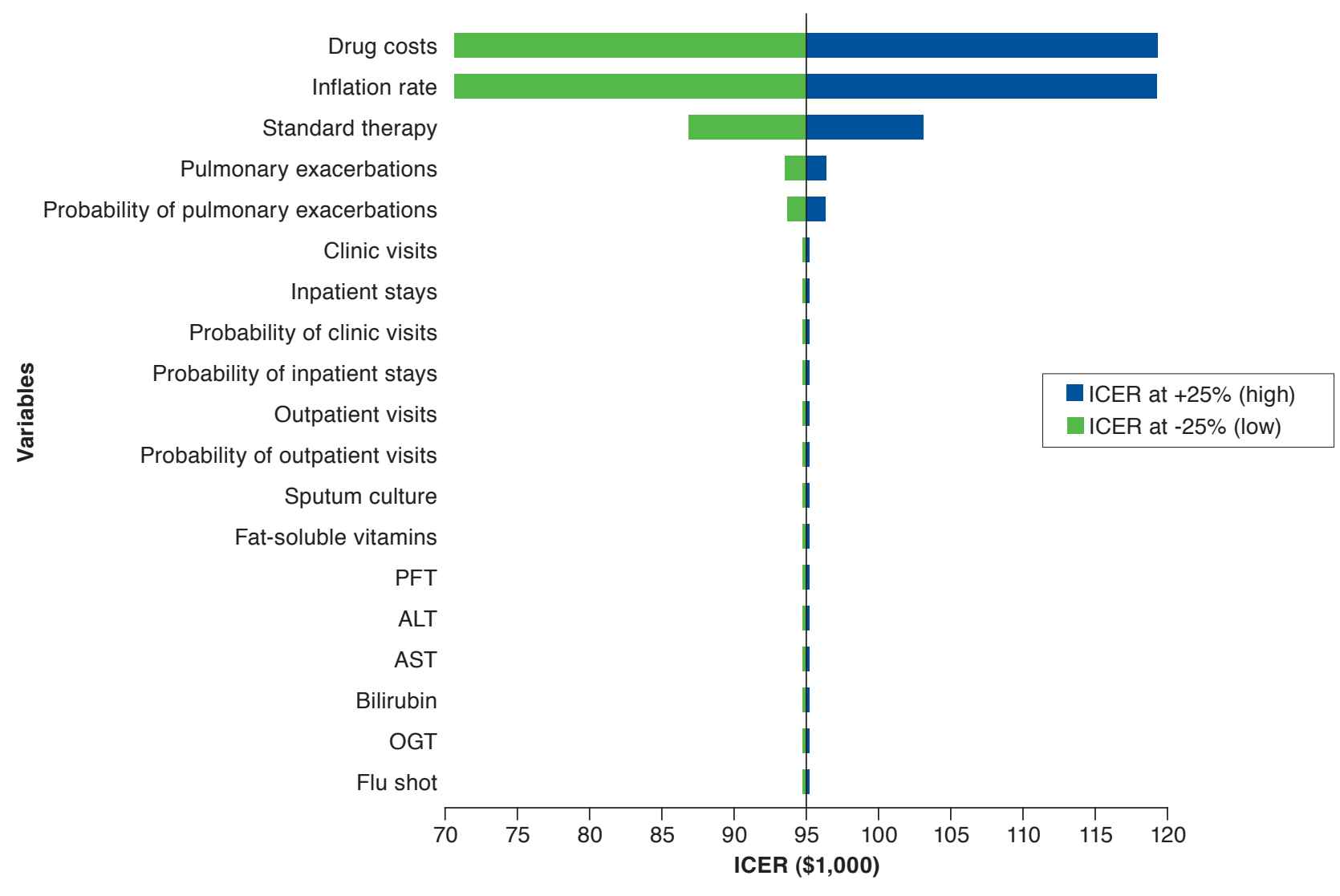

AST = aspartate aminotransferase test; $A L T=$ alanine aminotransferase test; $I C E R=$ incremental cost-effectiveness ratio; PFT = pulmonary lung function test; OGT = oral glucose tolerance test.

with the ICER ranging from $\$ 70,843$ to $\$ 119,054$, cost of standard therapy with the ICER ranging from $\$ 87,004$ to $\$ 103,029$, cost to treat pulmonary exacerbations with the ICER ranging from $\$ 93,660$ to $\$ 96,373$, and probability of occurrence of pulmonary exacerbations with the ICER ranging from $\$ 93,791$ to $\$ 96,241$. The ICER of lumacaftor/ivacaftor was not affected by varying the cost of other variables and probabilities of occurrence of medical events. Figure 1 presents a tornado plot for the one-way sensitivity analysis of cost-effectiveness of lumacaftor/ ivacaftor. Input ranges were plotted to determine the effect of different variables on the ICER.

\section{Base-Case Analysis: Budget Impact of Lumacaftor/Ivacaftor}

The prevalence of CF in the entire U.S. population was estimated at $0.00897 \%$, with an extrapolated estimate of CF prevalence in those aged 12 years or above calculated as 24,609 of 274,062,018 (0.00897\%; Appendix B, available in online article). ${ }^{29,30}$ Similarly, the prevalence of CF in a hypothetical plan population of 1 million was estimated at 89.70 . Of the
$89.70,77.50(86.4 \%)$ were estimated to have the F508del mutation, with $46.1 \%$ of those qualifying with homozygous F508del mutation, leading to the target population estimation of $35.72 .{ }^{20}$ Using these estimates, the cost of therapy per patient for standard therapy alone before the inclusion of lumacaftor/ ivacaftor was $\$ 113,735$ and after the inclusion of lumacaftor/ ivacaftor on the formulary was $\$ 379,780$. Hence, the total budget impact due to inclusion of the drug on the formulary was $\$ 266,046$. Based on a hypothetical plan population of 1 million individuals, the PMPM cost was $\$ 0.02$, with the PTMPM cost of $\$ 620.67$ based on 35.72 treated population.

\section{Sensitivity Analysis: Budget Impact of Lumacaftor/Ivacaftor} After a $\pm 25 \%$ change in the cost of lumacaftor/ivacaftor, the drug had the PTMPM cost ranging from $\$ 410.75$ to $\$ 830.59$. Similarly, lumacaftor/ivacaftor was sensitive to change in inflation rate with the PTMPM ranging from $\$ 462.76$ to $\$ 777.69$, change in cost to treat pulmonary exacerbations with the PTMPM cost ranging from $\$ 611.81$ to $\$ 629.53$, and probability 


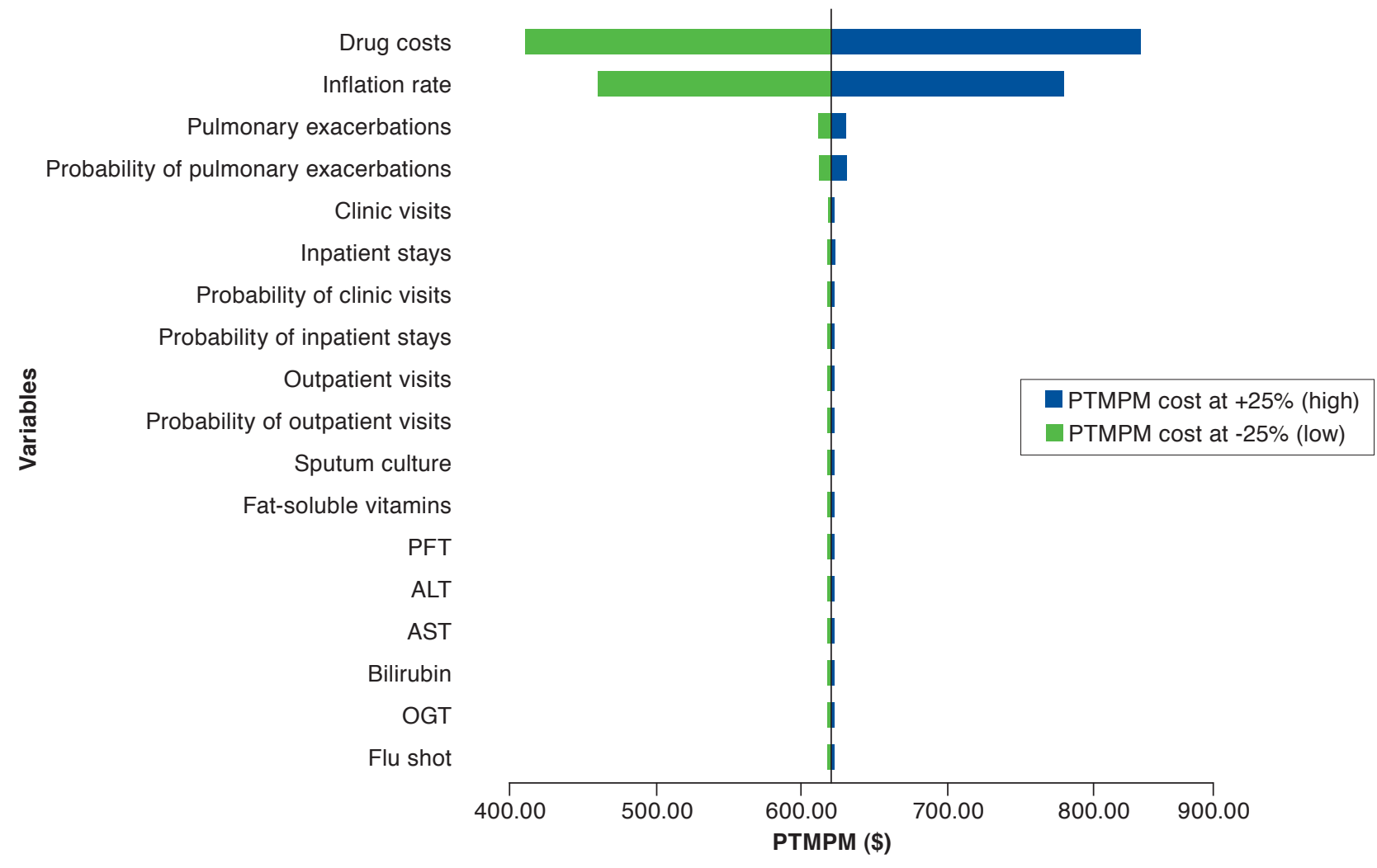

$A L T=$ alanine aminotransferase test; $A S T=$ aspartate aminotransferase test; $P F T=$ pulmonary lung function test; $O G T=$ oral glucose tolerance test; PTMPM = per treated member per month.

of occurrence of pulmonary exacerbations with the PTMPM cost ranging from $\$ 611.52$ to $\$ 628.67$. The PTMPM cost of lumacaftor/ivacaftor was not affected by varying the cost of other variables and probabilities of occurrence of medical events. Figure 2 presents the tornado plot for the one-way sensitivity analysis of the budget impact of lumacaftor/ivacaftor. Input ranges were plotted to determine the effect of different variables on the PTMPM cost.

\section{Discussion}

CFTR modulator therapies have revolutionized the treatment paradigm for CF patients. With changing health care policies and reimbursement patterns, managing health care costs is critical. Insurance companies are increasingly demanding health economic evidence to support their formulary decisions. The CFTR modulator therapies have good clinical effectiveness, including an increase in $\mathrm{FEV}_{1} \%$ predicted values, improvement in revised CF questionnaire (CFQR) scores, improved body mass index, and reduction in pulmonary exacerbation events. These regimens, therefore, have the potential to increase median life expectancy and delay the occurrence of death. But, the annual cost of lumacaftor/ivacaftor is estimated to be $\$ 270,000$, and its overall affordability is not established. Given the unmet need, this study was conducted to understand its value for the cost in the U.S. health care system. As there are no published studies that have assessed the cost-effectiveness of lumacaftor/ivacaftor in a U.S. population, the results of this study can be discussed within the context of published international studies. The HTA conducted by the NCPE of Ireland evaluated lumacaftor/ivacaftor compared with the standard of care using a lifetime simulation model and estimated an ICER of $€ 369,141$ per QALY (U.S. \$415,350 in 2016). ${ }^{15}$ The major differences between this study and NCPE HTA were the source of data and choice of clinical endpoints. The model inputs for NCPE HTA were provided by a single nonpublic source, whereas this study was conducted as a part of academic research and model inputs were taken from public sources like the CMS Physician Fee Schedule, RED BOOK, and published literature. The NCPE HTA used QALY as a clinical outcome, whereas this study used $\mathrm{FEV}_{1} \%$ predicted as the clinical 
endpoint. QALY is preferred in a cost-utility analysis or a CEA when utilities for each transition state and long-term clinical effectiveness is known. But this study used $\mathrm{FEV}_{1} \%$ predicted because the long-term clinical effectiveness and the transition states possible after treatment initiation were not known at the time this study was conducted.

While cost-effectiveness thresholds in the form of cost per QALY are commonly used in the United Kingdom, Canada, and Australia to make new treatment approval decisions, there are isolated cases of its use in the United States. ${ }^{31}$ Different value frameworks in the United States, list different thresholds, but generally, treatments under $\$ 50,000$ per QALY gained are considered of "high value." Recently, the World Health Organization used the country's gross domestic product to set thresholds, which, for the United States, would have translated as "intermediate value" or "somewhat cost-effective" for thresholds within the range of $\$ 100,000$ to $\$ 150,000$ and "economically unattractive" or "low value" over $\$ 150,000$ per QALY. 32 Given that the ICER of lumacaftor/ivacaftor in this study was $\$ 95,016$ per $\mathrm{FEV}_{1} \%$ predicted, there is a need for payers to further examine the treatment in light of the value frameworks, including their budget needs and patient needs.

The CFTR modulator therapies are the only available disease-modifying therapies that have the potential to increase median life expectancy in $\mathrm{CF}$ patients. A similar scenario was observed for nintedanib and pirfenidone, first-in-class medications approved recently for the treatment of idiopathic pulmonary fibrosis. A CEA conducted from a U.K. payer perspective concluded that the lifetime ICER was $£ 145,310$ per QALY gained (U.S. $\$ 183,762.35$ per QALY in 2016) for nintedanib and $£ 172,198$ per QALY gained (U.S. \$217,564.54 per QALY in 2016) for pirfenidone compared with placebo. ${ }^{33}$ These disease-modifying therapies have shown significant differences versus placebo in improving acute exacerbation events and lung function decline. Hence, the possible increase in life expectancy with newer treatments may be a justification for accepting the higher ICERs in the United States. The results of the sensitivity analysis suggest that after varying the data input ranges, lumacaftor/ivacaftor therapy was stable to drug monitoring schedules but were sensitive to variability in cost of the drug, inflation rates, cost of standard therapy, and cost and probability of pulmonary exacerbations. However, the ICER of lumacaftor/ivacaftor was most likely to be influenced by change in the cost of the drug, standard therapy, and cost to treat pulmonary exacerbations.

A BIA is an essential part of a comprehensive economic assessment of a new treatment and is increasingly being requested by payers, along with a CEA, before formulary approval or reimbursement. The purpose of a BIA is to estimate the financial consequences of adoption and diffusion of a new health care intervention within a specific health care setting to understand any resource constraints. There were no previous studies in the United States that assessed the affordability of lumacaftor/ivacaftor. However, the BIA conducted by NCPE on a target population of 505 patients assessed an annual cost for lumacaftor/ ivacaftor at $€ 158,306$ (U.S. $\$ 178,122$ in 2016) and a 1 -year budget impact of $€ 78,378,536$ (U.S. $\$ 92,736,703$ in 2016) and concluded that the budget impact due to lumacaftor/ ivacaftor was significant with an associated opportunity cost. ${ }^{15}$

In comparison, this study's 1-year budget impact of lumacaftor/ivacaftor per member was $\$ 264,000(\$ 0.022 \times 1$ million $\times 12$ months) and per treated member was $\$ 266,044$ $(\$ 620.67 \times 35.72 \times 12)$, similar to the findings of NCPE, indicating a significant economic impact on the U.S. health care budget. Although the Institute for Clinical and Economic Review has published an annual threshold of $\$ 904$ million for estimated potential budget impact for each individual new molecular entity for 2016 within the United States, ${ }^{34}$ decisions regarding BIAs are made purely by the payers, and most of these decisions are contingent on the balance between the increase in PMPM and PTMPM costs and the need of individual patients. The results of the sensitivity analysis suggest that even after varying the data input ranges, lumacaftor/ivacaftor therapy was stable to most of the variables except for the cost of the drug, inflation rates, and pulmonary exacerbation.

Lumacaftor/ivacaftor is listed as a specialty tier, requires prior authorization, or is not listed under some formularies, indicating reduced access to the CF population..$^{35-37}$ Lumacaftor/ivacaftor is covered under Medicare Part D with a typical copay range from $\$ 6,014$ to $\$ 24,057$. Because CF is more prevalent in children and young adults, Medicare might not cover the largest proportion of CF patients. However, with an average monthly copay of $\$ 1,252$ for those who are covered under Medicare, patients reach the donut hole (coverage gap of $\$ 3,310$ in 2016) in the third month, which makes the patients responsible for a major part of their prescription costs. Once patients go beyond the donut hole (also called catastrophic coverage, $\$ 4,850$ in 2016), Medicare once again covers most of the drug costs. ${ }^{38}$

The findings of this analysis can be used by payers and policymakers to predict how a change in the use of lumacaftor/ ivacaftor can affect the trajectory of CF-related spending. These results can be used for budget planning, forecasting, and calculating the effect of health technology changes on premiums in health insurance schemes. This study has major implications for payers and providers in their selection of cost-effective and affordable treatments for patients with CF.

\section{Limitations}

Although our study is one of the few studies to conduct a pharmacoeconomic evaluation of lumacaftor/ivacaftor, there are some limitations to consider. First, there were no existing comparator 
drugs, since lumacaftor/ivacaftor is indicated for CF patients with homozygous F508del mutations, hence, the evaluations involved comparisons against placebo or standard therapy.

Second, standard therapy available in the market consisted of maintenance therapies; our study was restricted to the standard therapy mentioned in the published clinical trials of lumacaftor/ivacaftor, which did not include drugs used to treat complications due to $\mathrm{CF}$.

Third, cost inputs and probabilities of events used in the models were obtained from multiple sources, including Medicare reimbursements, Healthcare Bluebook, and published literature. Although one-way sensitivity analyses were conducted to vary input ranges, the resulting estimates of any modeling study need to be treated with some degree of caution.

Fourth, it was not possible to develop dynamic models consisting of transition state probabilities, since lumacaftor/ ivacaftor was not approved until 2015. Long-term clinical effectiveness and events after the use of the drug were not available at the time of this study.

Fifth, the clinical efficacy of both treatment groups identified from the TRAFFIC and TRANSPORT trials may not be a true representation of the U.S. CF population, since the trials were multinational and the study population was identified from about 90 sites. This study conducted only univariate sensitivity analyses to test for uncertainty in the model parameters. Finally, the analyses assumed 100\% adherence to treatment and stable prevalence of CF across 2015-2016, which may differ from real-world scenarios.

\section{Conclusions}

In patients with CF, lumacaftor/ivacaftor has demonstrated better clinical effectiveness compared with placebo alongside significant costs of therapy. However, novel CFTR modulator therapies offer new disease-modifying benefits to patients with CF and may be considered an appropriate alternative to the existing standard therapy. Although the analysis focused on clinical outcomes and cost, decision makers selecting lumacaftor/ivacaftor may be influenced by multiple factors including adverse event profile, ease of administration, clinical outcomes, and coverage policies. The results may not be generalizable beyond a l-year time horizon, especially if serious adverse events associated with lumacaftor/ivacaftor play a role in influencing the overall cost. The results of this study will assist payers in making better decisions regarding the choice of lumacaftor/ivacaftor over a 1-year period for patients with cystic fibrosis and with homozygous F508del mutation.

\section{Authors}

PRATYUSHA VADAGAM, MS; KHALID M. KAMAL, MPharm, PhD; JORDAN R. COVVEY, PharmD, PhD, BCPS; and VINCENT GIANNETTI, PhD, Division of Pharmaceutical, Administrative and Social Sciences, Duquesne University School of Pharmacy, Pittsburgh, Pennsylvania. KUMAR MUKHERJEE, PhD, Pharmacy Practice, Philadelphia College of Osteopathic Medicine, Suwanee, Georgia.

AUTHOR CORRESPONDENCE: Khalid M. Kamal, MPharm, PhD, Professor, Division of Pharmaceutical, Administrative and Social Sciences, Duquesne University School of Pharmacy, 600 Forbes Ave., 418B Mellon Hall, Pittsburgh, PA 15282. Tel.: 412.396.1926;

E-mail:kamalk@duq.edu.

\section{DISCLOSURES}

No outside funding supported this study. Covvey and Kamal have received research funding from Novartis Pharmaceuticals. Covvey, Giannetti, and Kamal have received research funding from the College of Psychiatric and Neurologic Pharmacists. Kamal serves as a consultant to the Lynx Group (Cranbury, NJ) and Manticore Consulting Group (Scottsdale, AZ). Mukherjee has nothing to disclose.

A related poster abstract was presented at the AMCP Managed Care \& Specialty Pharmacy Annual Meeting; March 27-30, 2017; Denver, CO.

\section{REFERENCES}

1. National Heart, Lung, and Blood Institute. Facts about cystic fibrosis November 1995. Available at: https://www.cdc.gov/scienceambassador/documents/cystic-fibrosis-fact-sheet.pdf. Accessed July 24, 2018.

2. Cystic Fibrosis Foundation. About cystic fibrosis. Available at: https:// www.cff.org/What-is-CF/About-Cystic-Fibrosis/. Accessed July 24, 2018.

3. British Columbia, Healthlink BC. How cystic fibrosis affects digestion and the pancreas. May 4, 2017. Available at: https://www.healthlinkbc.ca/healthtopics/ug1494. Accessed August 27, 2018

4. Mayo Clinic. Cystic fibrosis: diagnosis \& treatment. October 13, 2016. Available at: http://www.mayoclinic.org/diseases-conditions/cystic-fibrosis/ diagnosis-treatment/diagnosis/dxc-20211900. Accessed July 24, 2018.

5. Cystic Fibrosis Foundation. Considerations for the use of lumacaftor and ivacaftor fixed dose combination oral tablets (Orkambi) for the management of persons with cystic fibrosis and two F508del CFTR mutations. August 28, 2015. Available at: https://www.cff.org/PDF-Archive/Orkambi-Whitepaper/. Accessed July 24, 2018.

6. Gee L, Abbott J, Conway SP, Etherington C, Webb AK. Quality of life in cystic fibrosis: the impact of gender, general health perceptions and disease severity. J Cyst Fibros. 2003;2(4):206-13.

7. Becker CC, Clements K, DeLong K, Harrow B, O'Sullivan A. PRS18 economic burden of cystic fibrosis in the US: costs of care by disease severity and age [abstract]. Value Health. 2011;14(7):A490.

8. Walgreens Specialty Pharmacy and Cystic Fibrosis Services. Impact of Walgreens Connected Care Cystic Fibrosis program on adherence and quality of life for enrolled CF patients. 2016. Available at: https://www.walgreens.com/images/adaptive/si/pdf/WalgreensConnectedCareCysticFibrosis WhitePaper.pdf. Accessed July 24, 2018.

9. Kalydeco (ivacaftor) tablets. Vertex Pharmaceuticals. January 2012. Available at: http://www.accessdata.fda.gov/drugsatfda_docs/label/2012/ 203188lbl.pdf. Accessed July 24, 2018. 
10. Orkambi (lumacaftor/ivacaftor) tablets, for oral use. Vertex Pharmaceuticals. July 2015. Available at: http://pi.vrtx.com/files/uspi_lumacaftor_ivacaftor. pdf. Accessed July 24, 2018.

11. Vertex Pharmaceuticals. A study of lumacaftor in combination with ivacaftor in cystic fibrosis subjects aged 12 years and older who are homozygous for the F508del-CFTR mutation (TRAFFIC). ClinicalTrials.gov Identifier NCT01807923. August 31, 2015. Available at: https://clinicaltrials. gov/ct2/show/NCT01807923?term=NCT01807923\&rank=1. Accessed July 24, 2018

12. Vertex Pharmaceuticals. A study of lumacaftor in combination with ivacaftor in cystic fibrosis subjects aged 12 years and older who are homozygous for the F508del-CFTR mutation (TRANSPORT). ClinicalTrials.gov Identifier NCT01807949. September 27, 2016. Available at: https://clinicaltrials.gov/ct2/show/NCT01807949?term=NCT01807949\&rank=1. Accessed August 28, 2018.

13. Elborn JS, Ramsey BW, Boyle MP, et al. Efficacy and safety of lumacaftor/ ivacaftor combination therapy in patients with cystic fibrosis homozygous for Phe508del CFTR by pulmonary function subgroup: a pooled analysis. Lancet Respir Med. 2016;4(8):617-26.

14. Truven Health Analytics, IBM Watson Health. Micromedex RED BOOK. Wholesale acquisition cost of lumacaftor/ivacaftor. Database. January 18, 2017. Available at: https://truvenhealth.com/Products/Micromedex/ProductSuites/Clinical-Knowledge/RED-BOOK. Accessed July 24, 2018.

15. National Centre for Pharmacoeconomics. Cost-effectiveness of lumacaftor/ivacaftor (Orkambi) for cystic fibrosis in patients aged 12 years and older who are homozygous for the F508del mutation in the CFTR gene. June 2016. Available at: http://www.ncpe.ie/wp-content/uploads/2015/12/ Website-summary-orkambi.pdf. Accessed July 25, 2018.

16. Semedo D. NICE says cost of Orkambi for cystic fibrosis is too high for low benefit. Cystic Fibrosis News Today. June 23, 2016. Available at: https:// cysticfibrosisnewstoday.com/2016/06/23/cost-of-cystic-fibrosis-treatmenttoo-high-for-benefit-offered/. Accessed July 25, 2018.

17. Wainwright CE, Elborn JS, Ramsey BW, et al. Lumacaftor-ivacaftor in patients with cystic fibrosis homozygous for Phe508del CFTR. N Engl J Med. 2015;373(3):220-31.

18. Konstan MW, McKone EF, Moss RB, et al. Assessment of safety and efficacy of long-term treatment with combination lumacaftor and ivacaftor therapy in patients with cystic fibrosis homozygous for the F508del-CFTR mutation (PROGRESS): a phase 3, extension study. Lancet Respir Med. 2017;5(2):107-18

19. Bang H, Zhao H. Cost-effectiveness analysis: a proposal of new reporting standards in statistical analysis. J Biopharm Stat. 2014;24(2):443-60.

20. Cystic Fibrosis Foundation. Patient registry: annual data report 2013. 2014. Available at: https://www.cff.org/2013_CFF_Patient_Registry_ Annual_Data_Report.pdf. Accessed July 25, 2018.

21. Centers for Medicare \& Medicaid Services. 2016 physician fee schedule. Available at: https://www.cms.gov/apps/physician-fee-schedule/search/ search-criteria.aspx. Accessed July 25, 2018.

22. Healthcare Bluebook. Available at: https://healthcarebluebook.com/. Accessed March 20, 2017.

23. Rubin-Cahill JL, Thayer S, Watkins A, Wagener J, Hodgkins P, Schechter MS. 190 frequency and costs of pulmonary exacerbations and association with \% predicted $\mathrm{FEV}_{1}$ in patients with cystic fibrosis. J Cyst Fibros. 2015;14(Suppl 1):S106.

24. Bureau of Labor Statistics. Consumer Price Index. All urban consumers 2007-2017. Available at: https://www.bls.gov/cpi/. Accessed July 25, 2018.

25. Strunk S, Koerner P, Craft Z, Miller R. Retrospective analysis of the medication utilization, clinical outcomes, and economic impact of patients treated with lumacaftor/ivacaftor (Orkambi) for cystic fibrosis. Poster presented at: AMCP Managed Care \& Specialty Pharmacy Annual Meeting 2016; April 19-22, 2016; San Francisco, CA.
26. Kobelt G, Berg J, Lindgren P, Jonsson B, Stawiarz L, Hillert J. Modeling the cost-effectiveness of a new treatment for MS (natalizumab) compared with current standard practice in Sweden. Mult Scler. 2008;14(5):679-90.

27. Bell C, Graham J, Earnshaw S, Oleen-Burkey M, Castelli-Haley J, Johnson K. Cost-effectiveness of four immunomodulatory therapies for relapsing-remitting multiple sclerosis: a Markov model based on long-term clinical data. J Manag Care Pharm. 2007;13(3):245-61. Available at: https:// www.jmcp.org/doi/10.18553/jmcp.2007.13.3.245

28. Chiao E, Meyer K. Cost effectiveness and budget impact of natalizumab in patients with relapsing multiple sclerosis. Curr Med Res Opin. 2009;25(6):1445-54.

29. Cystic Fibrosis Foundation. Patient registry: annual data report 2015. 2016. Available at: https://www.cff.org/Our-Research/CF-PatientRegistry/2015-Patient-Registry-Annual-Data-Report.pdf. Accessed July 25, 2018.

30. ChildStats.gov. Number of children (in millions) ages 0-17 in the United States by age, 1950-2016 and projected 2017-2050. Available at: https://www. childstats.gov/americaschildren/tables/popl.asp. Accessed July 25, 2018.

31. Dubois RW. Cost-effectiveness thresholds in the USA: are they coming? Are they already here? J Comp Eff Res. 2016;5(1):9-11.

32. Marseille E, Larson B, Kazi DS, Kahn JG, Rosen S. Thresholds for the cost-effectiveness of interventions: alternative approaches. Bull World Health Organ. 2015;93(2):118-24.

33. Rinciog C, Watkins M, Chang S, et al. A cost-effectiveness analysis of nintedanib in idiopathic pulmonary fibrosis in the UK. Pharmacoeconomics. 2017;35(4):479-91.

34. Institute for Clinical and Economic Review. Overview of the ICER value assessment framework and update for 2017-2019. Available at: https://icerreview.org/wp-content/uploads/2018/03/ICER-value-assessment-frameworkupdate-FINAL-062217.pdf. Accessed July 25, 2018.

35. ConnectiCare. 2017 prior authorization (PA) criteria. Available at: https://www.connecticare.com/medicare/pdf/Medicare_Part_D_Clinical_ Prior_Authorizations.pdf. Accessed August 27, 2018.

36. Kaiser Permanente. Prior authorization medications requiring reviewcriteria for use. Updated October 15, 2015. Available at: http://www.providers.kaiserpermanente.org/info_assets/cpp_ga/pdfs/ga_qrmguidelines_october_2015.pdf. Accessed July 25, 2018.

37. Priority Health. Priority Health Medicare prior authorization criteria (Part D): Orkambi. Updated March 2016. Available at: http://www.priorityhealth.com/medicare/once-you-enroll/drug-coverage/restrictions/ /media/ documents/medicare/2016/2016-drug-pa-pdfs/2016-orkambi-pa-criteria. pdf. Accessed July 25, 2018.

38. GoodRx. Orkambi Medicare coverage. Available at: https://www.goodrx. com/orkambi/medicare-coverage. Accessed July 25, 2018.

39. Proventil HFA (albuterol sulfate) inhalation aerosol. Schering. 2012. Available at: http://www.accessdata.fda.gov/drugsatfda_docs/label/2012/ 020503s046lbl.pdf. Accessed July 25, 2018.

40. Tobi (tobramycin) inhalation solution. Novartis. October 2015. Available at: https://www.pharma.us.novartis.com/sites/www.pharma.us.novartis. com/files/tobi.pdf. Accessed July 25, 2018.

41. Pulmozyme (dornase alfa) inhalation solution. Genentech. Revised January 2018. Available at: https://www.gene.com/download/pdf/pulmozyme_prescribing.pdf. Accessed July 25, 2018.

42. Enderby B, Doull I. Hypertonic saline inhalation in cystic fibrosis-salt in the wound, or sweet success? Arch Dis Child. 2007;92(3):195-96.

43. Flonase (fluticasone propionate) nasal spray, $50 \mathrm{mcg}$. GlaxoSmithKline. July 2003. Available at: http://www.accessdata.fda.gov/drugsatfda_docs/lab el/2004/20549slr016,20548slr020,20121slr030_flonase_lbl.pdf. Accessed July 25, 2018. 
44. Quest Diagnostics. Aspartate aminotransferase (AST). Available at: http://www.questdiagnostics.com/testcenter/BUOrderInfo. action?tc $=822 \&$ labCode=SEA. Accessed July 25, 2018.

45. Healthcare Bluebook. Transferase (AST) (SGOT). Available at: https:// healthcarebluebook.com/page_ProcedureDetails.aspx?id=954\&dataset=lab\& $\mathrm{g}=$ Transferase $+($ AST $)+($ SGOT $)$. Accessed July 25, 2018.

46. Quest Diagnostics. Alanine aminotransferase (ALT). Available at: http://www.questdiagnostics.com/testcenter/BUOrderInfo. action?tc $=823 \&$ labCode=DAL. Accessed July 25, 2018.

47. Healthcare Bluebook. Alanine Aminotransferase (ALT). Available at: https://www.healthcarebluebook.com/page_ProcedureDetails.aspx?cftid= Ll169\&g=Alanine+Aminotransferase+(ALT) \&directsearch=true. Accessed August 27, 2018,

48. Quest Diagnostics. Bilirubin, total and direct, neonatal. Available at: http://www.questdiagnostics.com/testcenter/BUOrderInfo. action?tc $=6631 \&$ labCode=SEA. Accessed July 25, 2018.

49. Healthcare Bluebook. Bilirubin total. Available at: https://healthcarebluebook.com/page_ProcedureDetails.aspx?dataset=lab\&id=967\&g=Bilirub in\%20Total\&directsearch=true. Accessed July 25, 2018.

50. Quest Diagnostics. Culture, sputum/lower respiratory. Available at: http://www.questdiagnostics.com/testcenter/BUOrderInfo.action?tc= 4556\&labCode=SEA. Accessed July 25, 2018.

51. Prices should be readily available to save hospitals money. KevinMD.com. June 9, 2011. Available at: http://www.kevinmd.com/blog/2011/06/pricesreadily-save-hospitals-money.html. Accessed July 25, 2018.

52. Micro Direct. CPT codes for spirometry. January 2004. Available at: https://mdspiro.com/image/data/PDFs/Micro\%20Direct\%20-\%20 Spirometry\%20Billing\%20Information\%202014.pdf. Accessed July 25, 2018.

53. Centers for Medicare \& Medicaid Services. 2017 physician fee schedule. Spirometry. Available at: https://www.cms.gov/apps/physician-fee-schedule/ search/search-results. aspx? $\mathrm{Y}=1 \& \mathrm{~T}=0 \& \mathrm{HT}=0 \& \mathrm{CT}=3 \& \mathrm{Hl}=94010 \& \mathrm{M}=5$. Accessed July 25, 2018.

54. Centers for Medicare \& Medicaid Services. Medicare Part B immunization billing: seasonal influenza virus, pneumococcal, and hepatitis B. January 2018. Available at: https://www.cms.gov/Outreach-and-Education/Medicare-LearningNetwork-MLN/MLNProducts/downloads/qr_Immun_bill.pdf. Accessed August 28, 2018.

55. Healthcare Bluebook. Flu (seasonal) vaccine. Available at: https:// healthcarebluebook.com/page_ProcedureDetails.aspx?dataset $=$ cc\& $\&$ id $=16 \& g=$ Flu\%20(seasonal)\%20Vaccine\&directsearch=true. Accessed July 25, 2018.

56. Quest Diagnostics. Vitamin A (retinol). Available at: http://www.questdiagnostics.com/testcenter/BUOrderInfo. action?tc=921\&labCode $=$ SJC. Accessed July 25, 2018.

57. Quest Diagnostics. Vitamin D, 25-hydroxy, total, immunoassay. Available at: http://www.questdiagnostics.com/testcenter/BUOrderInfo. action?tc=17306\&labCode=AMD. Accessed July 25, 2018.
58. Quest Diagnostics. Vitamin E (tocopherol). Available at: http://www. questdiagnostics.com/testcenter/BUOrderInfo. action?tc=931\&labCode=SJC. Accessed July 25, 2018.

59. Quest Diagnostics. Vitamin K. Available at: http://www.questdiagnostics. com/testcenter/TestDetail.action?ntc=36585. Accessed July 25, 2018.

60. Healthcare Bluebook. Vitamin A. Available at: https://healthcarebluebook.com/page_ProcedureDetails.aspx?cftid=L1425\&g=Vitamin\%20 A\&directsearch=true. Accessed July 25, 2018.

61. Healthcare Bluebook. Vitamin D 1 25-dihydroxy. Available at: https:// healthcarebluebook.com/page_ProcedureDetails.aspx?dataset=lab\&id=1576

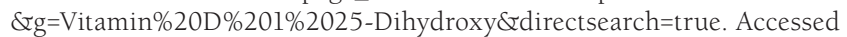
July 25, 2018.

62. Healthcare Bluebook. Tocopherol alpha (vitamin E). Available at: https:// healthcarebluebook.com/page_ProcedureDetails.aspx?dataset=lab\&id=1429 $\& g=$ Tocopherol\%20Alpha\%20(Vitamin\%20E)\&directsearch=true. Accessed July 25, 2018.

63. Healthcare Bluebook. Vitamin K. Available at: https://healthcarebluebook.com/page_ProcedureDetails.aspx?dataset=lab\&id=1430\&g=Vitam in\%20K\&directsearch=true. Accessed July 25, 2018.

64. Quest Diagnostics. Oral glucose tolerance test. Available at: https://www.questdiagnostics.com/testcenter/BUOrderInfo.action?tc= 10559\&labCode=PHP. Accessed August 28, 2018.

65. Healthcare Bluebook. Oral glucose tolerance test. Available at: https:// healthcarebluebook.com/page_ProcedureDetails.aspx?id=1501\&dataset=lab $\& g=$ Glucose+Tolerance+Test+(GTT). Accessed July 25, 2018.

66. HCPro. CMS replaces clinic E/M visit levels with single G-code. JustCoding News: Outpatient. January 8, 2014. Available at: http://www. hcpro.com/HIM-299741-8160/CMS-replaces-clinic-EM-visit-levels-withsingle-Gcode.html. Accessed July 25, 2018.

67. O'Sullivan AK, Sullivan J, Higuchi K, Montgomery AB. Health care utilization \& costs for cystic fibrosis patients with pulmonary infections. Manag Care. 2011;20(2):37-44.

68. Bradley JM, Blume SW, Balp M-M, Honeybourne D, Elborn JS. Quality of life and healthcare utilisation in cystic fibrosis: a multicentre study. Eur Respir J. 2013;41(3):571-77.

69. Vadagam P, Kamal KM, Mukherjee K, Covvey J, Giannetti V. Burden of illness in cystic fibrosis: a retrospective analysis of Medical Expenditure Panel Survey (MEPS) data. Poster presented at: International Society for Pharmacoeconomics and Outcomes Research Annual Meeting; May 24, 2017; Boston, MA.

70. Schlesinger R. The size of the U.S. and the world in 2016. U.S. News and World Report. January 5, 2016. Available at: https://www.usnews.com/opinion/blogs/robert-schlesinger/articles/2016-01-05/us-population-in-2016-according-to-census-estimates-322-762-018. Accessed July 25, 2018. 


\begin{tabular}{|c|c|c|c|c|c|c|c|}
\hline \multicolumn{8}{|c|}{ APPENDIX A Probabilities of Medical Events ${ }^{67}$} \\
\hline Variable & $\begin{array}{c}\text { Number } \\
\text { Considered for } \\
\text { Analysis }\end{array}$ & Discontinued & $\begin{array}{l}\text { Number with the } \\
\text { Event, } \mathrm{n}(\%)\end{array}$ & $\begin{array}{l}\text { Group Followed } \\
\text { Until (years) }\end{array}$ & Patient Years & Rate of Event & Probability \\
\hline Clinic visits & 1,064 & 0 & 862.0 (81.01) & 1 & 1,064 & 0.810 & $1-0.444=0.555$ \\
\hline Outpatient visits & 1,064 & 0 & $860.0(80.80)$ & 1 & 1,064 & 0.808 & $1-0.445=0.554$ \\
\hline Inpatient stays & 1,064 & 0 & $359.0(33.76)$ & 1 & 1,064 & 0.337 & $1-0.713=0.286$ \\
\hline Placebo $^{a}$ & 371 & 6 & $185.0(49.80)$ & 0.9 & 334 & $\begin{array}{c}185 / 334=0.55 \\
(182+6 / 2)^{b}\end{array}$ & $1-0.609=0.391$ \\
\hline Lumacaftor/ivacaftora $^{\mathrm{a}}$ & 369 & 17 & $140.5(38.00)$ & 0.9 & 332 & $\begin{array}{c}140.5 / 332=0.42 \\
(132+17 / 2)^{\mathrm{b}}\end{array}$ & $1-0.685=0.314$ \\
\hline
\end{tabular}

a Half considered to be followed until 24 weeks. ${ }^{17}$

${ }^{b}$ Total number with the event was calculated as the sum of those with the event and half of those who discontinued; patient years=number considered for analysis multiplied by the time followed; rate of event = number with event/patient years; probability $=1-e^{-r^{*} t}$ where $r=$ rate of medical event and $t=$ time horizon $(1$ year).

\section{APPENDIX B Target Population for Budget Impact Analysis of Lumacaftor/lvacaftor}

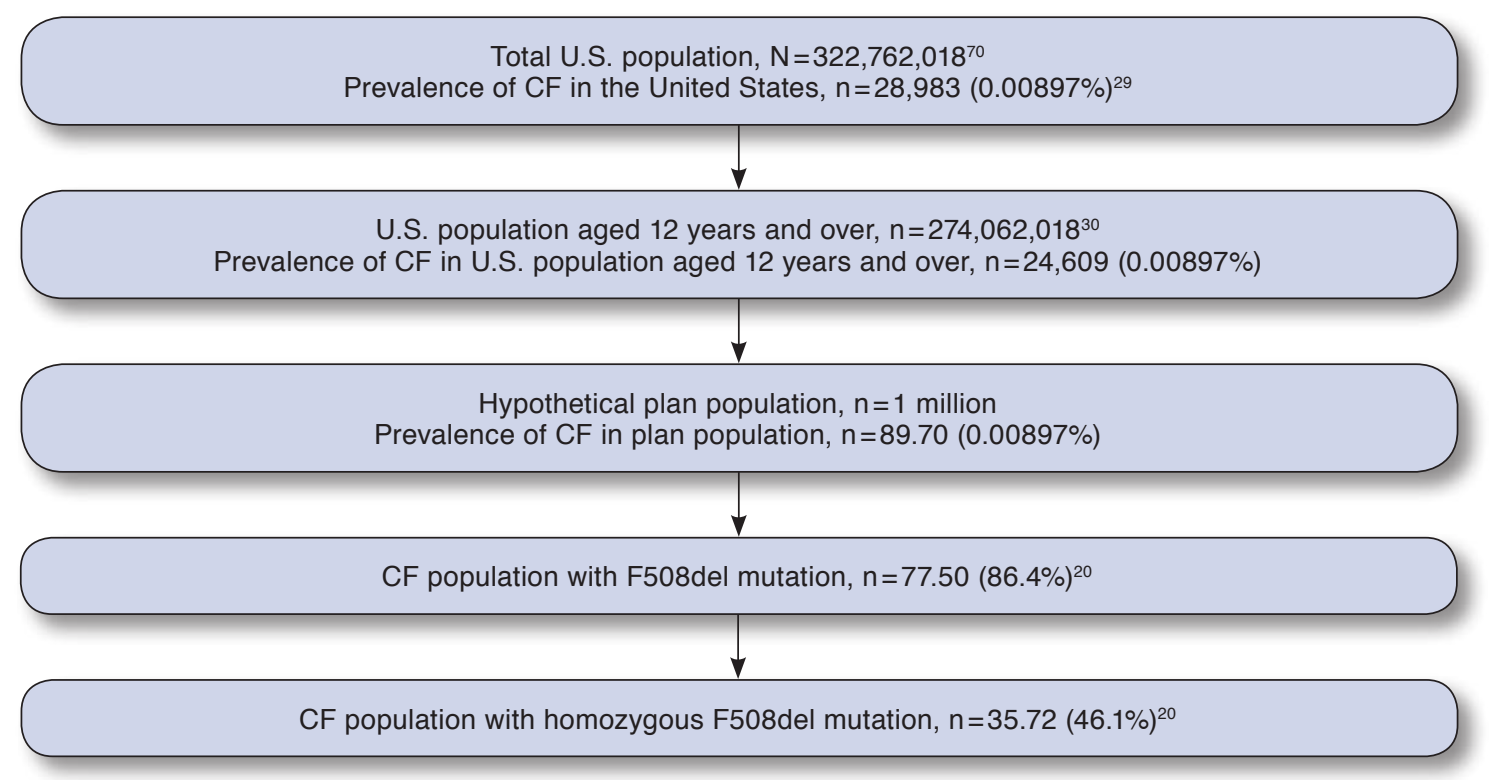

CF $=$ cystic fibrosis. 